\title{
Comparison of array comparative genomic hybridization and quantitative real-time PCR-based aneuploidy screening of blastocyst biopsies
}

\author{
Antonio Capalbo ${ }^{\star, 1,2}$, Nathan R Treff ${ }^{3,4}$, Danilo Cimadomo ${ }^{1,2}$, Xin Tao $^{3}$, Kathleen Upham ${ }^{3}$, \\ Filippo Maria Ubaldi ${ }^{1,2}$, Laura Rienzi ${ }^{1,2}$ and Richard T Scott $\mathrm{Jr}^{3,4}$
}

Comprehensive chromosome screening (CCS) methods are being extensively used to select chromosomally normal embryos in human assisted reproduction. Some concerns related to the stage of analysis and which aneuploidy screening method to use still remain. In this study, the reliability of blastocyst-stage aneuploidy screening and the diagnostic performance of the two mostly used CCS methods (quantitative real-time PCR (qPCR) and array comparative genome hybridization (aCGH)) has been assessed. aCGH aneuploid blastocysts were rebiopsied, blinded, and evaluated by qPCR. Discordant cases were subsequently rebiopsied, blinded, and evaluated by single-nucleotide polymorphism (SNP) array-based CCS. Although $81.7 \%$ of embryos showed the same diagnosis when comparing aCGH and qPCR-based CCS, 18.3\% (22/120) of embryos gave a discordant result for at least one chromosome. SNP array reanalysis showed that a discordance was reported in ten blastocysts for aCGH, mostly due to false positives, and in four cases for qPCR. The discordant aneuploidy call rate per chromosome was significantly higher for aCGH $(5.7 \%)$ compared with qPCR $(0.6 \% ; P<0.01)$. To corroborate these findings, 39 embryos were simultaneously biopsied for aCGH and qPCR during blastocyst-stage aneuploidy screening cycles. 35 matched including all 21 euploid embryos. Blinded SNP analysis on rebiopsies of the four embryos matched qPCR. These findings demonstrate the high reliability of diagnosis performed at the blastocyst stage with the use of different CCS methods. However, the application of aCGH can be expected to result in a higher aneuploidy rate than other contemporary methods of CCS.

European Journal of Human Genetics (2015) 23, 901-906; doi:10.1038/ejhg.2014.222; published online 29 October 2014

\section{INTRODUCTION}

There are now multiple randomized controlled trials (RCTs), which have demonstrated meaningful improvements in the clinical outcomes of IVF with the incorporation of comprehensive chromosome screening (CCS) of aneuploidy ${ }^{1-4}$ even if additional studies are necessary to determine which patients may or may not benefit from CCS. ${ }^{5}$ However, the RCT alone is incapable of determining the clinical negative predictive value of a CCS aneuploidy diagnosis, as the embryos diagnosed as abnormal are not transferred. Without such an evaluation, there remains a risk that reproductively competent embryos are erroneously discarded owing to technical errors (false positives) in the screening method itself.

For example, a previous study demonstrated that nearly $60 \%$ of blastocysts were chromosomally normal in four sections and for all 24 chromosomes despite a cleavage stage fluorescent in situ hybridization (FISH) aneuploidy diagnosis. ${ }^{6}$ This indicates that FISH is poorly predictive of aneuploidy as a result of a high rate of false positives when used on single cells. Several other methods of chromosome screening have demonstrated poor predictive value of an aneuploidy diagnosis based on finding normalcy later in development. ${ }^{7,8}$ However, a more relevant assessment of the negative predictive value of a screening method is the ultimate clinical outcome after transfer of an embryo that would have been predicted as aneuploidy.
Indeed, such a study has been conducted and demonstrated that an aneuploidy diagnosis by a specific CCS method provided $96 \%$ predictive value of a negative clinical outcome. ${ }^{9}$ Given that this single-nucleotide polymorphism (SNP) array-based CCS method has also been extensively evaluated for preclinical accuracy ${ }^{10}$ and clinical efficacy, ${ }^{4,11}$ it represents a legitimate benchmark to evaluate new methods of CCS.

For example, quantitative real-time PCR (qPCR)-based CCS was recently developed to provide a more rapid and inexpensive method than array-based methods such as SNP arrays and array comparative genomic hybridization (aCGH). qPCR was evaluated for preclinical accuracy using cell lines with known abnormalities and embryos with prior array-based diagnoses. ${ }^{12}$ The results indicated $98-99 \%$ consistency with expected karyotypes of cell lines and embryos. In addition to having published preclinical accuracy, this method has also demonstrated clinical efficacy in two RCTs. ${ }^{2,3}$

In contrast, aCGH CCS has not published preclinical accuracy using single cells isolated from cell lines with previously established karyotypes or blastomeres from embryos with prior validated CCS diagnoses. Instead, studies claiming validation of aCGH have used concordance with FISH, ${ }^{13,14}$ despite the fact that FISH-based technology has failed to demonstrate clinical validity in multiple RCTs. ${ }^{15}$ A recent RCT of aCGH indicated improved clinical efficacy in

${ }^{1}$ GENERA, Centers for Reproductive Medicine, Rome, Naples, Umbertide, Marostica, Italy; ${ }^{2}$ GENETYX, Molecular Genetics Laboratory, Marostica, Vicenza, Italy; ${ }^{3}$ Reproductive Medicine Associates of New Jersey, Basking Ridge, NJ, USA; ${ }^{4}$ Division of Reproductive Endocrinology, Department of Obstetrics, Gynecology and Reproductive Science, Robert Wood Johnson Medical School, Rutgers University, New Brunswick, NJ, USA

*Correspondence: Dr A Capalbo, GENERA Centre for Reproductive Medicine, Clinica Valle Giulia, Via G. De Notaris 2/B, 00197 Rome, Italy. Tel: +0039 063269791; Fax: +0039 0632697979; E-mail: capalbo@generaroma.it

Received 12 May 2014; revised 11 September 2014; accepted 16 September 2014; published online 29 October 2014 
good-prognosis patients. ${ }^{1}$ However, the reported aneuploidy rates were unusually high $(45 \%)$ for patients with a mean maternal age of 32 years. The detriment of overdiagnosis of aneuploidy in younger good-prognosis patients may not be manifested within the first attempts at embryo transfer. However, in patients of advanced maternal age or patients with single-gene disorders performing also an aneuploidy screening cycle, the impact of false-positive aneuploidy may be more severe. These issues only reiterate the importance of determining the clinical negative predictive value and preclinical accuracy of new CCS methodologies before routine implementation. Such information is currently not available for aCGH.

As an alternative to performing a nonselection study of aCGH, the present study will provide a comparison of aCGH and qPCR, by using preponderance of evidence from three contemporary methods of CCS performed on the same embryos. Importantly, the use of three methods of analysis provides the added benefit of controlling for mosaicism within the embryo as both $\mathrm{qPCR}$ and aCGH are given the same chance of being influenced by biological variation within the embryo. Furthermore, in this study different contemporary CCS methods have been blindly used to perform 24-chromosome copy number analysis on multiple trophectoderm (TE) biopsies from the same embryos, providing the first unbiased assessment of the reliability of the blastocyst-stage aneuploidy screening approach.

\section{MATERIALS AND METHODS}

\section{Study design and sample specification}

This is a prospective double-blinded observational study performed between October 2012 and December 2013. The analysis was focused on TE biopsies (Figure 1) to compare qPCR-based 24-chromosome screening with the most widely used aCGH method in the preimplantation genetic screening (PGS) field. Blastocysts from 45 patients underwent TE biopsy at GENERA and aneuploidy screening by 24 sure and BlueGnome (Cambridge, UK) aCGH at a commercial reference laboratory. Blastocysts (Supplementary Table 1) showing copy number aneuploidies by aCGH were warmed, re-biopsied, and blinded for evaluation by 4 -h qPCR. Following the second biopsy, blastocysts were cryopreserved according to Italian law. Embryos showing discordant qPCR results were subsequently warmed again, rebiopsied, blinded, and evaluated by a third method of CCS based on SNP arrays. When SNP arrays confirmed one of the initial methods, the remaining method was considered to have given a discordant result. The impact of biological variation due to mosaicism in the embryo is specifically minimized given the known random distribution in the different TE samples from the same embryo. ${ }^{16}$ That is, mosaicism will similarly affect the two CCS methods under investigation. As this is an observational study where only aneuploid blastocysts were selected for reanalysis, the assessment of aCGH and qPCR diagnostic performances was performed on a per chromosome basis.

To corroborate initial findings and to potentially include in the analysis also euploid embryos, 39 blastocysts were simultaneously analyzed using aCGH and qPCR. Following diagnosis, discordant cases were blinded and reanalyzed using SNP array on a second TE biopsy and results were evaluated as previously described.

The Ethics Committee of Clinica Valle Giulia approved the study and signed informed consent for the chromosome reanalysis of aneuploid blastocysts was obtained from all patients who underwent a PGS cycle at GENERA, Reproductive Medicine Center. IRB approval for analysis of discarded material from the second phase was obtained from western IRB.

\section{IVF procedures}

During ICSI/PGS cycles fertilized oocytes were sequentially cultured in separate $35-\mu 1$ microdrops (Sage In-Vitro Fertilization, Inc., Trumbull, CT, USA) up to blastocyst stage (Day 5/6) in a humidified atmosphere containing 5\% $\mathrm{O}_{2}$ and $6 \% \mathrm{CO}_{2}$. Expanding and expanded blastocysts underwent biopsy of TE cells and cryopreservation on day 5. Cavitating morulas were transferred to a fresh individual 35-ml drop of blastocyst medium (Quinn's Advantage Blastocyst

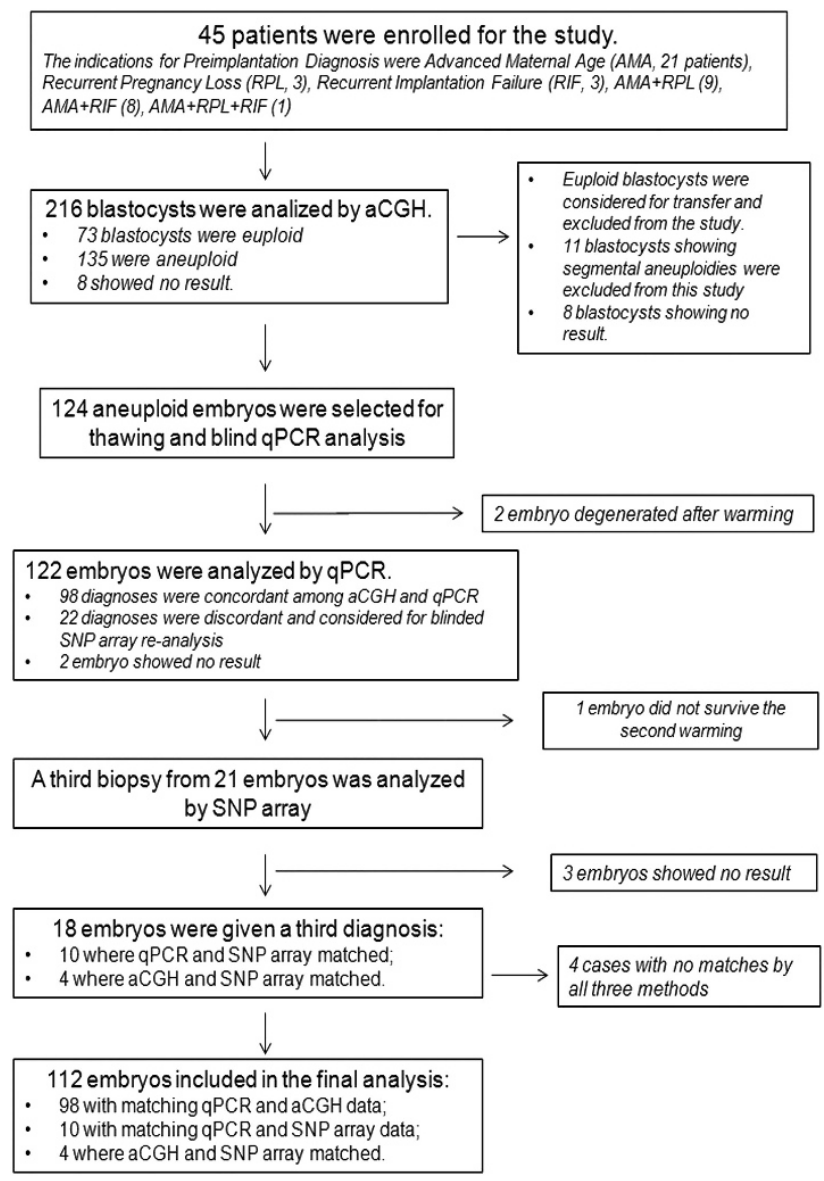

Figure 1 Flow chart of phase 1 preclinical validation of aCGH and qPCRbased aneuploidy screening of trophectoderm biopsies.

Medium, Cooper Surgical, Trumbull, CT, USA) with 15\% serum protein substitute (Quinns Advantage Serum Protein Substitute, Cooper Surgical, Trumbull, CT, USA) and biopsy was attempted $24 \mathrm{~h}$ later on day 6 or $48 \mathrm{~h}$ later on day 7. Blastocyst quality was assessed immediately before TE biopsy, defined according to the criteria presented by Gardner and Schoolcraft. ${ }^{17}$ Biopsied blastocysts were vitrified according to the protocol described by Nagy et al. ${ }^{18}$ Aneuploid blastocysts were selected for reanalysis and thawed for a second TE biopsy. All the TE biopsy procedures were performed on the heated stage of a Nikon IX-70 microscope (Nikon instruments, Campi Bisenzio FI, Italy), equipped with micromanipulation tools, in dishes prepared with three droplets of $10 \mathrm{ml}$ of HEPES-buffered medium (Sage In-Vitro Fertilization, Inc.) overlaid with pre-equilibrated mineral oil. A diode laser (Research Instruments, Cornwall, UK) was used to assist the opening of a 10-20 $\mu \mathrm{m}$ hole in the zona pellucida. The biopsy procedure was performed as previously described. ${ }^{19} 5-10$ TE cells were aspirated into the TE biopsy pipette (Research Instruments) followed by laser-assisted removal of the target cells from the body of the embryo. TE rebiopsies on aneuploid embryos were carried out following the same protocols used for TE biopsies during clinical PGS cycles. All TE biopsies were washed in sterile phosphate-buffered saline (PBS) or in hypotonic solution (depending on the CCS method) in a laminar flow cabinet to avoid any contamination of the sample, placed in microcentrifuge tubes to be further processed for CCS.

\section{Genetic testing methods}

Each method of CCS was performed using protocols that are in clinical use by each of the respective laboratories. The initial diagnosis obtained by aCGH was performed by a commercial reference laboratory according to the manufacturer's protocol (BlueGnome) and as previously described. ${ }^{20}$ The second 
analysis was performed using qPCR-based CCS as previously described. ${ }^{12}$ SNP array-based CCS was performed as previously described. ${ }^{10}$ qPCR-based CCS has undergone multiple phases of validation including the evaluation of $\mathrm{QPCR}$ results of cells from cell lines, which had already had conventional G-banding. The same approach was initially used for the SNP array method used in the present study. ${ }^{10}$ In addition, qPCR results were compared with those obtained by SNP array-based CCS on the same embryos. While the results were highly concordant, they did not impact the way in which qPCR or SNP array is performed and therefore there is no reason to expect that $\mathrm{qPCR}$ would be any more similar to SNP array than to aCGH, as they are all independent methods.

\section{Statistical analysis}

Numerical and categorical data are reported as mean and proportion with $95 \%$ confidence interval (CI). Consistency between the three CCS methods used on the same embryos was evaluated at the level of individual chromosome copy numbers for the entire 24 chromosomes of each sample tested and for the overall diagnosis of aneuploidy or euploidy. Discordance was reported when a CCS method did not match with the other two. In particular, discordant aneuploidy call rate was defined as aneuploidy detected for a specific chromosome from one CCS method but not confirmed from the other two CCS methods. Discordant euploidy call was reported when a normal chromosomal segregation was detected from one CCS method but the other two CCS methods reported the same aneuploidy for that chromosome. The diagnostic sensitivity and specificity of qPCR and aCGH based on concordance between the two CCS methods were measured on a per chromosome basis as the proportion of actual positives, which are consistently identified as such, and the proportion of negatives, which are consistently identified. Continuous data and categorical variables are presented as mean and percentage frequency with 95\% CI, respectively. A Fisher's exact test was used to assess differences between categorical variables. Alpha was set at 0.05 .

\section{RESULTS}

One hundred and twenty-four aneuploid blastocysts were selected from 45 patients who underwent a single PGS cycle with aCGH-based CCS (Figure 1). Detailed information about patients basal characteristics and PGS cycle outcomes are reported in Table 1. One hundred and twenty two $(98.4 \%$; 122/124; 95\% CI 94.3-99.8) blastocysts survived the first warming procedure and after re-expansion were biopsied for a TE sample and blinded for qPCR analysis. Two samples

\section{Table 1 Baseline characteristics of patients involved in the study and PGS cycles outcome}

\begin{tabular}{|c|c|}
\hline Number of patients & 45 \\
\hline \multicolumn{2}{|l|}{ Indication for PGS } \\
\hline AMA & $21(46.6 \%)$ \\
\hline RPL & $3(6.7 \%)$ \\
\hline RIF & $3(6.7 \%)$ \\
\hline$A M A+R P L$ & $9(20 \%)$ \\
\hline$A M A+R I F$ & $8(17.8 \%)$ \\
\hline $\mathrm{AMA}+\mathrm{RPL}+\mathrm{RIF}$ & $1(2.2 \%)$ \\
\hline Female age (SD; range) & $39.0( \pm 3.2 ; 30.3-43.3)$ \\
\hline Previous miscarriages (SD; range) & $0.9( \pm 1.2 ; 0-3)$ \\
\hline Previous IVF cycles (SD; range) & $2.0( \pm 1.9 ; 0-8)$ \\
\hline MII oocytes retrieved (SD; range) & $11.0( \pm 4.2 ; 5-24)$ \\
\hline Mean blastocyst per cycle (SD; range) & $5.0( \pm 2.3 ; 1-13)$ \\
\hline Biopsied blastocysts & 216 \\
\hline$\%$ day $5(N ; 95 \% \mathrm{Cl})$ & $56.5 \%(122 / 216 ; 49.6-63.2)$ \\
\hline$\%$ day $6(N ; 95 \% \mathrm{Cl})$ & $43.5 \%(94 / 216 ; 36.8-50.4)$ \\
\hline Aneuploidy rate $(\mathrm{N} ; 95 \% \mathrm{Cl})$ & $64.9 \%(135 / 208 ; 36.8-50.4)$ \\
\hline Single or double aneuploid $(N ; 95 \% \mathrm{Cl})$ & $89.5 \%(111 / 124 ; 82.7-94.3)$ \\
\hline Complex aneuploid $(\mathrm{N} ; 95 \% \mathrm{Cl})$ & 10.5\% (13/124; 5.7-17.3) \\
\hline
\end{tabular}

Abbreviations: $\mathrm{Cl}$, confidence interval; PGS, preimplantation genetic screening. gave no result following $\mathrm{qPCR}$ and were excluded from subsequent analysis. Interpretable qPCR results were obtained in 98.4\% (120/122; 95\% CI 94.2-99.8) of the TE samples. Although 81.7\% (98/120; 95\% CI 73.6-88.1) of embryos showed the same diagnosis when comparing aCGH and qPCR-based full karyotype analysis, $18.3 \%$ (22/120; 95\% CI 11.9-26.4) of embryos were discordant for at least one chromosome. Blastocysts showing a discordant result were warmed again and a third TE biopsy was performed on the 21 embryos surviving the second warming $(95.4 \% ; 21 / 22$; $95 \%$ CI 77.2-99.9) for blinded SNP array reanalysis. SNP array reanalysis of the 21 embryos yielded a conclusive diagnosis on 18 cases (85.7\%; 95\% CI 66.7-96.9). In four of these embryos $(3.3 \%$; 4/120; 95\%CI 0.9-8.3) all three CCS methods did not match when considering the full karyotype (Table 2) and were interpreted as mosaic aneuploid embryos. In 10 embryos (8.9\%; 10/112; 95\% CI 4.4-15.8) an inconsistent diagnosis was reported for aCGH, mostly due to discordant aneuploidy calls for aCGH. SNP array reanalysis revealed 10 discordant aneuploidy calls and 2 discordant euploidy calls at the single chromosome level by aCGH in these 10 embryos (embryo number 5, 16, 30, 81, 102, 110, 3, 11, 22, and 45; Table 2). These errors resulted in a discordant diagnosis of aneuploidy in the original aCGH diagnosis for six embryos $(5.4 \%$; 6/112; 95\% CI 2.0-11.3), where euploidy was diagnosed by both qPCR and SNP array. Five of these six discordant embryos were originally diagnosed by aCGH as single aneuploid, whereas one was diagnosed as double aneuploid (the profile plots of three of them are shown in Figure 2). An inconsistent diagnosis was reported for $\mathrm{qPCR}$ in the remaining four cases $(3.6 \% ; 4 / 112 ; 95 \%$ CI $0.1-8.9)$. Three discordant euploidy and one discordant aneuploidy were observed at the individual chromosome level. However, none of the qPCR discordances at the individual chromosome level translates in a diagnosis conversion from euploid to aneuploid or vice versa (0\%; 0/112; $95 \%$ CI $0.0-3.2$ ).

Looking at the single-chromosome level, 174 and 164 aneuploidies were reported by aCGH and $\mathrm{APCR}$, respectively. A consistent chromosome copy number diagnosis was observed in $99.4 \%$ (2561/2576; $95 \%$ CI 99.0-99.7) of the 2576 chromosomes analyzed. In this perchromosome analysis, the discordance aneuploidy call rate was significantly higher for aCGH $(5.7 \%$; 10/174; $95 \%$ CI $2.8-10.3)$ compared with qPCR $(0.6 \% ; 1 / 164,95 \%$ CI $0.0-3.3 ; P<0.01)$. The two CCS methods showed similar discordant euploidy call rates $(0.1 \% ; 2 / 2402$, 95\% CI $0.0-0.3$ for aCGH and $0.1 \% 3 / 2412$, 95\% CI 0.0-0.4 for qPCR, NS). qPCR and aCGH performed similarly in terms of sensitivity (98.2\%, 163/166, 95\% CI 94.8-99.6 vs $98.8 \%, 164 / 166$, 95\% CI 95.799.8, respectively, NS), whereas qPCR displayed a significantly higher specificity compared with aCGH $(99.9 \%, 2409 / 2410,95 \%$ CI $99.8-100$ vs $99.6 \%, 2412 / 2422$, 95\% CI 99.2-99.8, respectively, $P=0.01$ ).

To corroborate these findings, 39 embryos were simultaneously analyzed by aCGH and qPCR during blastocyst-stage CCS cycles (Supplementary Table 2). Thirty-five matched including all 21 euploid embryos. The four that did not match had two aneuploid chromosomes by aCGH, one by qPCR. Blinded SNP analysis on rebiopsies of the four embryos matched qPCR (Table 3).

Considering all embryos analyzed in the two phases of the study, a consistent chromosome copy number diagnosis from aCGH and qPCR on two different TE biopsies was reported for $99.86 \%$ (3468/3473; 95\% CI $0.99-1)$ of the chromosomes analyzed. The discordant aneuploidy call rate was significantly higher for aCGH (7.0\%; 14/201; 95\% CI 3.9-11.4) compared with qPCR (0.5\%; 1/188, 95\% CI 0-2.9). The two CCS methods showed similar discordant euploidy rates $(0.1 \% ; 3 / 3272,95 \%$ CI $0-0.3$ for aCGH and $0.1 \%$, $3 / 3285,95 \%$ CI $0-0.3$ for qPCR, NS). 
Table 2 Discordant cases between aCGH and qPCR with reassessment of diagnosis performed by a third trophectoderm biopsy and SNP array based CCS

\begin{tabular}{|c|c|c|c|}
\hline$E m b I D$ & $\mathrm{aCGH}$ & $q P C R$ & SNP array \\
\hline \multicolumn{4}{|c|}{ Embryo level discordant cases } \\
\hline 5 & Unbalanced male, -15 & Balanced male & Balanced male \\
\hline 16 & Unbalanced male, -8 & Balanced male & Balanced male \\
\hline 30 & Unbalanced female, +2 & Balanced female & Balanced female \\
\hline 81 & Unbalanced female, +22 & Balanced female & Balanced female \\
\hline 102 & Unbalanced female, $\mathrm{XO}$ & Balanced male & Balanced male \\
\hline 110 & Unbalanced male, $+14,+17$ & Balanced female & Balanced female \\
\hline \multicolumn{4}{|c|}{ Chromosome level discordant cases } \\
\hline 3 & Unbalanced male, $+16,-18,-21$ & Unbalanced male, $-18,-21,+22$ & Unbalanced male, $-18,-21,+22$ \\
\hline 11 & Unbalanced male, $-1,+16$ & Unbalanced male, +16 & Unbalanced male, +16 \\
\hline 22 & Unbalanced female, $-14,+22$ & Unbalanced female, -14 & Unbalanced female, -14 \\
\hline 45 & Unbalanced male, -22 & Unbalanced male, $-8,-22$ & Unbalanced male, $-8,-22$ \\
\hline 96 & Unbalanced male, $+12,+21$ & Unbalanced male, +21 & Unbalanced male, $+12,+21$ \\
\hline 2 & Unbalanced male, $+3,+20$ & Unbalanced male, +20 & Unbalanced male, $+3,+20$ \\
\hline 86 & Unbalanced female, - 15 & Unbalanced female, $-15,+22$ & Unbalanced female, - 15 \\
\hline 12 & Unbalanced male, $+15,+18$ & Unbalanced male, +18 & Unbalanced male, $+15,+18$ \\
\hline \multicolumn{4}{|c|}{ Cases discordant between all three CCS methods } \\
\hline 60 & Unbalanced female, -4 & Balanced female & Unbalanced female, -19 \\
\hline 119 & Unbalanced female, +21 & Unbalanced female, $+19+21$ & Balanced female \\
\hline 37 & Unbalanced male, +18 & Unbalanced male, +3 & Balanced male \\
\hline 78 & Unbalanced female, $-5+17-22$ & Unbalanced male, $X X Y+19$ & Unbalanced male, XXY \\
\hline
\end{tabular}

Abbreviations: aCGH, array comparative genome hybridization; CCS, comprehensive chromosome screening; qPCR, quantitative real-time PCR; SNP, single-nucleotide polymorphism.

\section{DISCUSSION}

This is the first prospective double-blinded observational study performed to evaluate the diagnostic performances of two contemporary technologies for CCS on TE biopsies in blastocyst-stage PGS cycles.

Because the presence of mosaicism in blastocysts as a result of postzygotic mitotic aneuploidy development represents a well-documented phenomenon that could contribute to biologic variation in blastocysts, ${ }^{5,16}$ the preponderance of evidence from three methods was used. Because mosaicism is randomly distributed to all three methods, one can justifiably attribute the observed overall differences in consistency between the methods to inherent inaccuracies in the technologies rather than to biological variation in the embryo. Furthermore, because each method was applied in its actual clinical setting, one can consider the results to be representative of actual clinical performance.

Overall, the analysis on a per chromosome basis of these blastocysts demonstrated a high concordance level between aCGH- and qPCRbased CCS, where $99.86 \%$ of chromosome predictions were consistent between the two methods applied on different TE biopsies from the same embryo. Even if the analysis of the whole TE sample cannot identify chromosomal non-disjunction events where a mix of trisomic and monosomic cells for the same chromosome is present, results from this study corroborate previous findings showing no major impact of chromosomal mosaicism and high diagnostic reliability when testing at the blastocyst stage. ${ }^{6,16}$ Only in four embryos all three methods did not match for all chromosomes, suggesting the presence of a true mosaicism in those samples and making it difficult to estimate the actual embryonic karyotype.

However, the SNP-based reanalysis of discordant cases highlighted a significantly higher discordant aneuploidy call rate from aCGH compared with qPCR-based CCS. Even if this is an end-point analysis and this study was not designed to investigate the reason behind a discordant chromosome aneuploidy prediction, some hypotheses can be formulated to explain the higher discordant aneuploidy detection rate from aCGH compared with $\mathrm{qPCR}$.

One possibility is the lack of chromosome-specific cutoffs for predicting aneuploidy from aCGH data. Instead, a universal cutoff is used to assign aneuploidy status to all chromosomes, possibly leading to false-positive diagnoses. Given that WGA (in this case Sureplex PCR-based WGA) may introduce chromosome-specific amplification bias owing to the unique GC content of each chromosome, there may be a need to incorporate chromosome-specific thresholds for diagnosis of aneuploidy by aCGH. In contrast, qPCR-based CCS uses chromosome-specific cutoffs established from actual data obtained from embryos with previously identified aneuploidies, thus providing the potential for more precise chromosome-specific diagnoses.

Another possible contribution to the higher aneuploidy rate observed for aCGH is related to the inherent limitations associated with competitive DNA hybridization. aCGH was originally developed to investigate the genetics of cancer. In this more conventional setting, the DNA is allowed to hybridize to the arrays for $\sim 72 \mathrm{~h}$, and is applied to relatively large quantities of DNA. In contrast, the protocol established for aCGH in the PGS setting is significantly shortened to $4 \mathrm{~h}$ and starts with far less than the recommended quantity of DNA. This represents a dramatic change from the conventional application of aCGH technology. In contrast, qPCR was originally established to allow for rapid completion $(\sim 1-2 \mathrm{~h})$ and when using limited starting material, making its adaptation to the PGS setting far less dramatic.

Avoiding false-positive results in the aneuploidy screening is of particular importance for patients of advanced maternal age or with single-gene disorders performing also PGS, where fewer reproductively competent or otherwise genetically normal embryos may be available per cycle. Furthermore, minimizing false-positive diagnoses is critical 
a

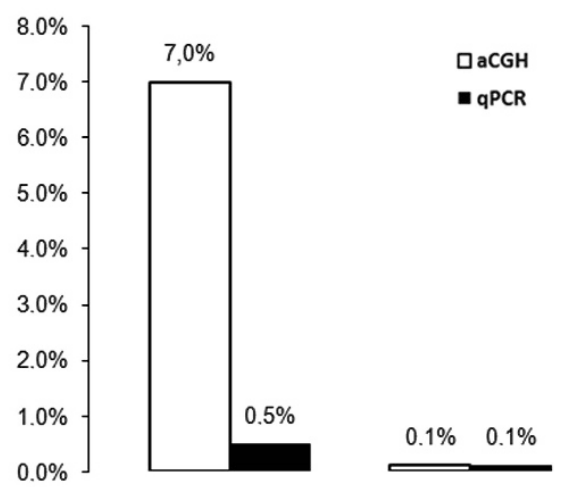

c

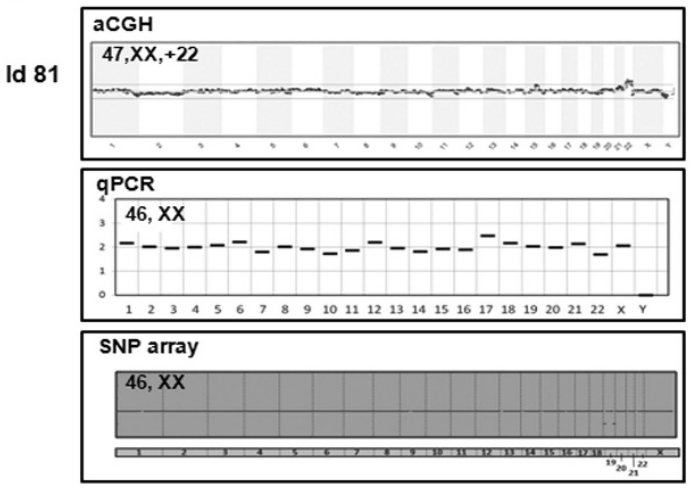

b

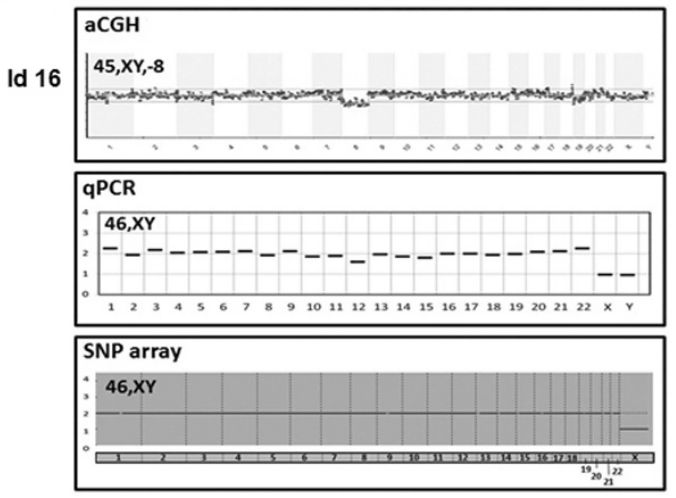

d

Id $31 \mathrm{~b}$

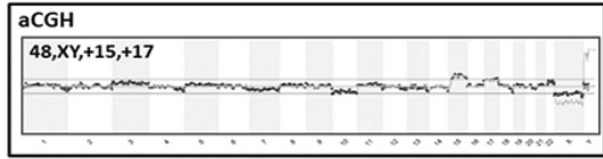

qPCR

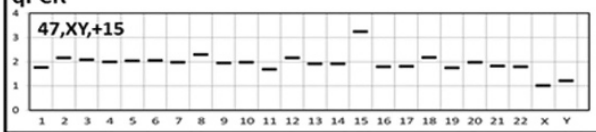

SNP array

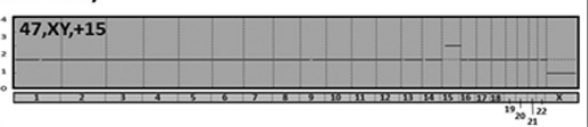

Figure 2 Profile plot comparison of some discordant cases between aCGH and qPCR with confirmation of diagnosis performed on a third biopsy and SNP array-based CCS. (a) False-positive and -negative chromosome error rates according to CCS methods under investigation. (b) Profile plots of aCGH showing a monosomy for the chromosome 18 for that embryo, shown to be normal by qPCR and SNP array-based CCS follow-up analysis. (c) aCGH profile plot consistent with a trisomy for chromosome 22 for that embryo, shown to be normal by qPCR and SNP array-based CCS follow-up analysis. (d) Profile plots of aCGH showing a double trisomy for chromosome 15 and 17 for that embryo, shown to be trisomy 15 by qPCR and SNP array-based CCS follow-up analysis.

Table 3 Four embryos simultaneously biopsied for aCGH and qPCR during blastocyst-stage CCS cycles and giving discordant diagnosis, then reassessed by aSNP-based CCS performed on a third trophectoderm biopsy in the second phase confirmation analysis

\begin{tabular}{lll}
\hline Emb ID & aCGH & SPCR \\
\hline $1 \mathrm{~b}$ & Unbalanced female, +19 & Unbalanced female, -11 \\
$22 \mathrm{~b}$ & Unbalanced female, $X X X,+16$ & Unbalanced female, +16 \\
$30 \mathrm{~b}$ & Unbalanced female, $-6,-9$ & Unbalanced female, -6 \\
$31 \mathrm{~b}$ & Unbalanced male, $+15,+17$ & Unbalanced male, +15 \\
\hline
\end{tabular}

Abbreviations: aCGH, array comparative genome hybridization; qPCR, quantitative real-time PCR; SNP, single-nucleotide polymorphism.

to obtaining the same pregnancy rate per cycle when comparing cumulative outcomes of PGS cycles to IVF cycles without PGS. Even if at a lower rate, there remains a risk that reproductively competent embryos are erroneously discarded owing to technical errors (false aneuploidy calls) in the screening method itself when poorly validated chromosomal screening methods are extensively applied in the clinical practice of PGS.

These results reiterate the importance of establishing the ability of an aneuploid diagnosis of new CCS methods to predict a negative clinical outcome. Until such time as aCGH methods are appropriately evaluated for their clinical negative predictive value, the present study represents the only available comparative evidence and indicates that the application of aCGH in the clinical setting can be expected to result in a higher aneuploidy rate than other contemporary and better validated methods of CCS.
Furthermore, owing to the high overall consistency of chromosome diagnosis reported in this study using different CCS methods on multiple TE biopsies from the same embryos, blastocyst-stage aneuploidy screening proved to be a highly reliable and effective approach for PGS.

\section{CONFLICT OF INTEREST}

The authors declare no conflict of interest.

1 Yang Z, Liu J, Collins GS et al: Selection of single blastocysts for fresh transfer via standard morphology assessment alone and with array CGH for good prognosis IVF patients: results from a randomized pilot study. Mol Cytogenet 2012; 5: 24.

2 Scott Jr RT, Upham KM, Forman EJ et al: Blastocyst biopsy with comprehensive chromosome screening and fresh embryo transfer significantly increases in vitro 
fertilization implantation and delivery rates: a randomized controlled trial. Fertil Steril 2013; 100: 697-703.

3 Forman EJ, Hong KH, Ferry KM et al: In vitro fertilization with single euploid blastocyst transfer: a randomized controlled trial. Fertil Steril 2013; 100: 100-107,e1.

4 Schoolcraft WB, Surrey E, Minjarez D, Gustofson RL, Scott Jr RT, Katz-Jaffe MG: Comprehensive chromosome screening (CCS) with vitrification results in improved clinical outcome in women $>35$ years: a randomized control trial. Fertil Steril 2012; 98: 718-724.

5 Bormann CL, Racowsky C: Is universal application of blastocyst biopsy with comprehensive chromosome screening for embryo selection ready for prime time? Fertil Steril 2013; 2: e5-e6.

6 Northrop LE, Treff NR, Levy B, Scott Jr RT: SNP microarray-based 24 chromosome aneuploidy screening demonstrates that cleavage-stage FISH poorly predicts aneuploidy in embryos that develop to morphologically normal blastocysts. Mol Hum Reprod 2010; 16: $590-600$.

7 Brezina PR, Benner A, Rechitsky S et al: Single-gene testing combined with single nucleotide polymorphism microarray preimplantation genetic diagnosis for aneuploidy: a novel approach in optimizing pregnancy outcome. Fertil Steril 2011; 95: e5-e8.

8 Geraedts J, Montag M, Magli MC et al: Polar body array CGH for prediction of the status of the corresponding oocyte. Part I: clinical results. Hum Reprod 2011; 26 3173-3180.

9 Scott RT Jr, Ferry K, Su J, Tao X, Scott K, Treff NR: Comprehensive chromosome screening is highly predictive of the reproductive potential of human embryos: a prospective, blinded, nonselection study. Fertil Steril 2012; 97: 870-875.

10 Treff NR, Su J, Tao X, Levy B, Scott Jr RT: Accurate single cell 24 chromosome aneuploidy screening using whole genome amplification and single nucleotide polymorphism microarrays. Fertil Steril 2010; 94: 2017-2021.

11 Schoolcraft WB, Treff NR, Stevens JM et al: Live birth outcome with trophectoderm biopsy, blastocyst vitrification, and single-nucleotide polymorphism microarray-based comprehensive chromosome screening in infertile patients. Fertil Steril 2011; 96 638-640.
12 Treff NR, Tao X, Ferry KM, Su J, Taylor D, Scott Jr RT: Development and validation of an accurate quantitative real-time polymerase chain reaction-based assay for human blastocyst comprehensive chromosomal aneuploidy screening. Fertil Steril 2012; 97: 819-824, e2.

13 Mir P, Rodrigo L, Mercader A et al: False positive rate of an arrayCGH platform for single-cell preimplantation genetic screening and subsequent clinical application on day-3. J Assist Reprod Genet 2013; 30: 143-149.

14 Gutierrez-Mateo C, Colls P, Sanchez-Garcia J et al: Validation of microarray comparative genomic hybridization for comprehensive chromosome analysis of embryos. Fertil Steril 2011; 95: 953-958.

15 Mastenbroek S, Twisk M, van der Veen F et al: Preimplantation genetic screening: a systematic review and meta-analysis of RCTs. Hum Reprod Update 2011; 17: 454-466.

16 Capalbo A, Wright G, Elliott $\mathrm{T}$ et al: FISH reanalysis of inner cell mass and trophectoderm samples of previously array-CGH screened blastocysts shows high accuracy of diagnosis and no major diagnostic impact of mosaicism at the blastocyst stage. Hum Reprod 2013; 8: 2298-2307.

17 Gardner DK, Schoolcraft WB. In vitro culture of human blastocysts. Jansen R, Mortimer D. Toward Reproductive Certainty: Fertility and Genetics Beyond. Carnforth, UK: Parthenon Publishing, 1999; 378-388.

18 Nagy ZP, Chang CC, Shapiro DB et al: Clinical evaluation of the efficiency of an oocyte donation program using egg cryo-banking. Fertil Steril 2008; 2: 520-526.

19 Capalbo A, Rienzi L, Cimadomo D et al: Correlation between standard blastocyst morphology, euploidy and implantation: an observational study in two centers involving 956 screened blastocysts. Hum Reprod 2014; 6: 1173-1181.

20 Capalbo A, Bono S, Spizzichino L et al: Sequential comprehensive chromosome analysis on polar bodies, blastomeres and trophoblast: insights into female meiotic errors and chromosomal segregation in the preimplantation window of embryo development. Hum Reprod 2013; 2: 509-518.

Supplementary Information accompanies this paper on European Journal of Human Genetics website (http://www.nature.com/ejhg) 